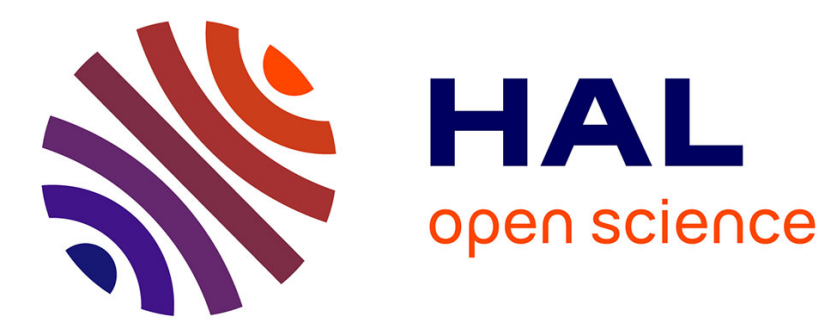

\title{
Flow and mixing by small intestine villi
}

Y. F. F Lim, Clément de Loubens, R. J. J Love, R. G. G Lentle, P. W. M. W M Janssen

\section{To cite this version:}

Y. F. F Lim, Clément de Loubens, R. J. J Love, R. G. G Lentle, P. W. M. W M Janssen. Flow and mixing by small intestine villi. Food and Function, 2015, 6 (6), pp.1787-1795. 10.1039/c5fo00285k . hal-02358110

\section{HAL Id: hal-02358110 https://hal.science/hal-02358110}

Submitted on 11 Nov 2019

HAL is a multi-disciplinary open access archive for the deposit and dissemination of scientific research documents, whether they are published or not. The documents may come from teaching and research institutions in France or abroad, or from public or private research centers.
L'archive ouverte pluridisciplinaire HAL, est destinée au dépôt et à la diffusion de documents scientifiques de niveau recherche, publiés ou non, émanant des établissements d'enseignement et de recherche français ou étrangers, des laboratoires publics ou privés. 


\title{
Flow and mixing by small intestine villi
}

\author{
Received 18th March 2015, Accepted 25th April 2015 in Food \& Function \\ First published on the web April 2015 \\ DOI: 10.1039/b000000x
}

Y.F. Lim, ${ }^{a}$ C. de Loubens, ${ }^{* b}$ R.J. Love, ${ }^{a}$ R.G. Lentle, ${ }^{a}$ and P.W.M. Janssen ${ }^{a}$

Flow and mixing in the small intestine are multi-scale processes. Flows at the scale of the villi (finger-like structures of $\approx 500$ $\mu \mathrm{m}$ length) are poorly understood. We developed a three-dimensional lattice-Boltzmann model to gain insight into the effects of villous movements and the rheology of digesta on flow, mixing and absorption of nutrients at the periphery of the intestinal lumen. Our model simulated the hydrodynamic consequences of villi movements that resulted from folding of the mucosa during longitudinal contractions. We found that cyclic approximation and separation of groups of villi generated laminar eddies at the edges of the group and augmented mass transfers in the radial direction between the inter-villous space and the intestinal lumen which improved the absorption of nutrients and mixing at the periphery of the lumen. This augmentation was greater with highly diffusible nutrients and with high levels of shear-thinning (pseudoplasticity) of the fluid. We compared our results with bulk flows simulations done by previous workers and concluded that villous movements during longitudinal contractions is a major radial mixing mechanism in the small intestine and increases mixing and absorption around the mucosa despite adverse rheology.

\section{Introduction}

Thorough mixing of enzymatic secretions with the contents of the lumen is essential for their efficient interaction, generation and absorption of nutrients at the mucosal surface of the small intestine. The major constraints to such mixing are the high viscosity and the non-Newtonian characteristics of the gastrointestinal content ${ }^{123}$ and the low velocity of their flow at the boundaries of the lumen, which together result in very low Reynolds numbers ${ }^{4}$. It is most likely that flows and transfers in the lumen should limit biochemical reactions during food digestion. Hence, it is essential to understand and model the mixing strategies developed by the digestive $\operatorname{tract}^{5}$ in an environment that precludes the formation of turbulence and for which the input of energy has to be minimal. Advances in high fidelity quantification of intestinal motility ${ }^{67}$, the application of chemical reactor theory 8910 to characterise mixing in exvivo segments of the small intestine ${ }^{1112}$ and the development of realistic models of intestinal fluid dynamics 41314151617 have enabled the effects of radial and longitudinal contractile activity on bulk flows and mixing in the intestinal lumen to be quantified ${ }^{5}$. Hence concerted circular and longitudinal contractions (i.e. peristaltic contractions) have been shown to both propel and mix the luminal content by generating radial and axial advective movements of digesta 1114151718 . In

\footnotetext{
a Institute of Food, Nutrition and Human Health, Massey University, Private Bag 11222, Palmerston North, New Zealand; E-mail: r.g.lentle@massey.ac.nz

${ }^{b}$ Aix Marseille Universit, CNRS, Centrale Marseille, IRPHE UMR 7342, 13384, Marseille, France; E-mail: deloubens@irphe.univ-mrs.fr
}

contrast, longitudinal contractions ${ }^{613}$ were shown to generate axial dispersion by the local stretching of the luminal contents with minimal axial propulsion. The latter process accelerated the diffusion of nutrients by shear dispersion whilst maximising residence time within the small intestine ${ }^{416}$. This process would mimic mixing by a small number of continuous stirred tank reactors in series ${ }^{12}$.

While it is generally accepted that such mixing strategies occur on a macroscopic level, flow and mixing at a microscopic level around the mucosa and villi, finger-like structures of around $500 \mu \mathrm{m}$ length, have not been rigorously studied, chiefly as the movements and the biomechanics of the villi have not, until recently, been quantified 192021 . Due to absence of these knowledges, it was commonly assumed that mass transfer at the periphery of the lumen was limited by a near static layer of fluid, termed the unstirred water layer (UWL), that was the rate-limiting step in absorption ${ }^{22} 23$. Various attempts to measure the thickness of the UWL lead to the contradictory hypothesis that movements of villi may influence mucosal absorption by generating stirring ${ }^{2425}$. The mucosa could be responsible for peripheral mixing, instead to be a resistance to absorption. More recently, generic twodimensional (2D) multi-scales models of flow in the gut ${ }^{26}$ and the characterization of mixing in the ex-vivo proximal duodenum of the rat ${ }^{12}$ gave support to the hypothesis that mixing at a microscopic scale in the periphery of the intestinal lumen could occur by itself, and is not limited by an hypothetic UWL, in contrast with industrial processes of transfer for which the interfaces are not mechanically active ${ }^{27}$.

Recent observations of the biomechanical behaviour of the 


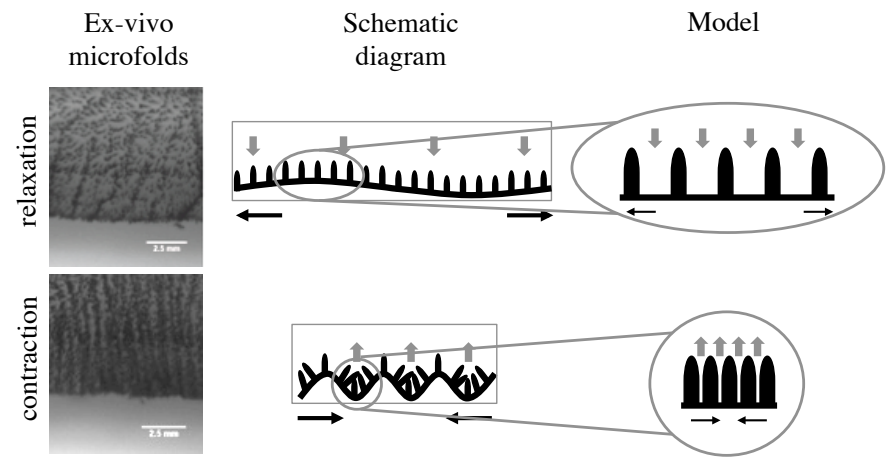

Fig. 1 Overview of the mechanism of formation of mucosal micro-folds during longitudinal muscular contractions (left and middle) and of the simplified geometry used for the lattice-Boltzmann model (right). Contraction of the longitudinal muscle caused cyclic mucosal micro-folding with approximation of villi in folds and separation on crests. The process of villus approximation in folds was represented in a single three-dimensional model in which the villi were maintained in parallel and moved only in the longitudinal direction. Adapted from ${ }^{19}$.

mucosa in ex-vivo everted segments of ileum of the possum ${ }^{19}$ have shown that short-lived radially disposed microfolds are formed in the mucosa during longitudinal contractions (figure 1) and confirmed that the mucosa has an active role on flow and mixing even at these microscopic scales. Further that the cyclic folding of the mucosal layers caused villi to crowd together, alternately pumping fluid into and out from the intervillous spaces. Preliminary $2 \mathrm{D}$ simulations demonstrated that these microfolds could generate peripheral stirring. Such a hypothesis is also supported by recent findings that villi are relatively rigid structures that cannot be deformed by physiological flow 21 .

The objective of this study was to analyse and quantify flow and mixing generated at the periphery of the small intestine during the formation of mucosal microfolds. We develop a three-dimensional (3D) model for Newtonian and nonNewtonian shear-thinning fluids (pseudo-plasticity) that focuses on the action of a one group of villi in a semi-infinite space, independent of flow phenomena around the central lumen. The model was based on lattice-Boltzmann methods $(\mathrm{LBM})$ as these are particularly useful for modeling flow in complex and moving geometries. As villus shapes vary between species and between different segments of the small intestine, as does solid volume content of digesta, mixing and absorption were simulated for a number of different geometrical configurations of villi and different degrees of shearthinning.

\section{Methods}

\subsection{Microscale motility of the small intestinal mucosa}

The formation of mucosal microfolds by the activity of longitudinal musculature caused groups of villi, in areas between

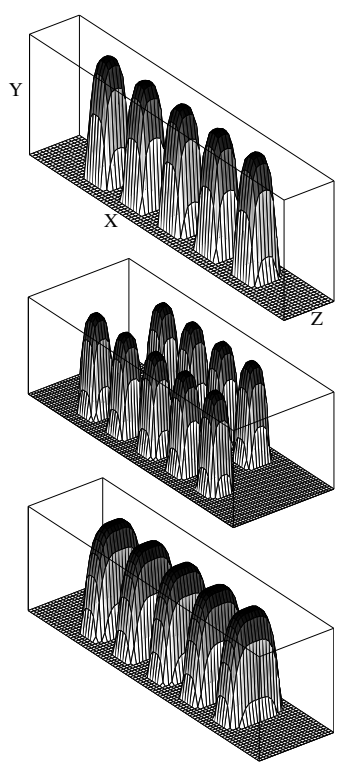

Fig. 2 The different sets of morphologies and spatial dispositions of villi that were used in the study: finger-like, aligned villi configuration (top); finger-like, staggered villi configuration (middle); and paddle-like, aligned villi configuration (bottom).

adjacent folds, to approximate and to separate during the subsequent relaxation and contraction. Likewise contraction caused the villi to separate on the tips of mucosal microfolds and to approximate during subsequent relaxation ${ }^{19}$ (figure 1). The three-dimensional (3D) model focused on a single group of villi between adjacent folds. The component villi were assumed to move only in the longitudinal direction. This simplification preserved the main characteristics of villous move- 
ment, i.e. grouping and separation, and made the model computationally tractable. The model focused on the effects of villi movements on flow and mixing independently of external flow phenomena in an unconfined configuration (i.e. no effects of external boundaries).

The number of villi in the model was limited in the longitudinal direction (i.e. $\mathrm{X}$ direction) and infinite in the radial direction (i.e. through periodic boundary conditions in the $\mathrm{Z}$ direction) (figure 2). The group of villi was enclosed in an infinite space in order to prevent any boundary effects influencing flow near the villi. The relative positions i.e. the spatial dispositions of villi were simulated in aligned and staggered configurations to reflect likely asymmetry in the placement of villi on the mucosa (figure 2 ). The $3 \mathrm{D}$ profile of the villi was modelled by a bi-dimensional fourth order polynomial function of $560 \mu \mathrm{m}$ length and $160 \mu \mathrm{m}$ base ${ }^{2021}$. In order to take into account the variety of shapes of small intestinal villi ${ }^{28}$, finger-like and paddle-like villi were modeled (figure 2 ). The component villi were assumed to be rigid structures as the limited reports of intrinsic motility in villi ${ }^{293031}$ did not quantify either their amplitude, frequency or direction and our recent study shows that they can be considered as a rigid structure under physiological flow ${ }^{21}$. The longitudinal velocity of individual villi was calculated by the integration of the strain rate $\dot{\varepsilon}\left(\right.$ in s $^{-1}$ ) in the longitudinal direction ${ }^{4}$. The cyclic variation of the longitudinal strain rate $\dot{\varepsilon}$ (in s${ }^{-1}$ ), i.e. the rate of local lengthening (if $\dot{\varepsilon} \leq 0$ ) or shortening (if $\dot{\varepsilon} \geq 0$ ), was represented by a sinusoidal function:

$$
\dot{\varepsilon}(t)=\dot{\varepsilon}_{\max } \sin \left(\frac{2 \pi}{T} t\right)
$$

where $\dot{\varepsilon}_{\max }$ is the amplitude $\left(=0.02 \mathrm{~s}^{-1}\right), t$ is the time (s) and $T$ the period of longitudinal activity $(=6.66 \mathrm{~s})^{419}$. Relaxation and contraction of the longitudinal muscular element in the model occurred at the time between 0 and $T / 2$ and $T / 2$ and $T$ respectively (figure 3 ).

\subsection{Flows, rheology and mixing}

Flow was modeled by the equation of mass and momentum for incompressible laminar flows

$$
\begin{gathered}
\frac{\partial u_{i}}{\partial x_{i}}=0 \\
\rho\left(\frac{\partial u_{i}}{\partial t}+u_{j} \frac{\partial u_{i}}{\partial x_{j}}\right)=\frac{\partial \tau_{i j}}{\partial x_{j}}-\frac{\partial p}{\partial x_{i}}
\end{gathered}
$$

where $i, j(=1,2,3), u_{i}, \rho, t, x_{i}, \tau_{i j}$ and $p$ are the indices of the system of coordinates, the velocity, the density, the time, the coordinate, the fluid stress tensor, and the pressure respectively. The stress tensor is related to the rate of strain $S_{i j}$

$$
\tau_{i j}=2 \eta(|\dot{\gamma}|) S_{i j}
$$

For Newtonian fluids, we have $\eta(|\dot{\gamma}|)=\eta_{0}$. For nonNewtonian shear-thinning fluids, the variation of the viscosity $\eta$ (in Pa.s) with the shear rate $|\dot{\gamma}|\left(\right.$ in s $^{-1}$ ) was modeled by the truncated version of the Carreau law for shear-thinning fluids

$$
\eta=\eta_{0}\left(1+\frac{|\dot{\gamma}|^{2}}{\dot{\gamma}_{c}^{2}}\right)^{\frac{n-1}{2}}
$$

where $\eta_{0}$ is the zero-shear viscosity, $\dot{\gamma}_{c}$ a critical shear rate beyond which the viscosity would decrease and $n$ is the powerlaw index of a value between 0 and 1 for shear-thinning fluids ( $n=1$ for Newtonian fluids). For value of $\dot{\gamma}_{c}$ around 10, this law is representative of biopolymers solutions as guar gum. When $\dot{\gamma}_{c}$ tends to 0 , the fluid behaviour is pseudo-plastic and representative of the rheological behaviour of the digesta in various compartments of the gut ${ }^{123}$.

The mixing and absorption of nutrients of diffusivity $D$ (in $\mathrm{m}^{2} / \mathrm{s}$ ) were modeled by the advection-diffusion equations

$$
\frac{\partial \mathrm{C}}{\partial t}+\mathbf{u} \nabla C=D \nabla^{2} C
$$

where $C$ is the concentration and $\mathbf{u}$ the flow velocity. We assumed that absorption was passive and not limited by epithelial permeability, i.e. the tracer was instantaneously absorbed when it reached the mucosa.

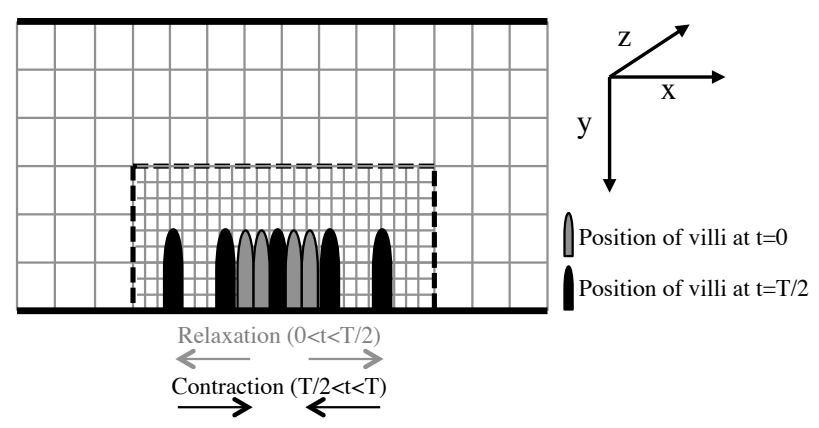

Fig. 3 Schema of computational simulation. A two dimensional slice of the three dimensional modelling environment is shown with the coarse and fine grid sizes used. The physical boundaries were placed sufficiently far from the fine grids to study flow in an infinite space. Periodic boundary conditions were used in the $\mathrm{Z}$ direction.

\subsection{Lattice-Boltzmann methods}

Flow and advection-diffusion equations were solved by LB methods, which are capable of modeling flow with moving 
boundaries on a Cartesian mesh without the remeshing routines that are generally used with other numerical methods ${ }^{32}$. A multi-grid strategy was developed to dismiss the influence of the boundaries on the flow in the region around the group of villi, i.e. to enable the villi to be modeled in an semi-infinite space (figure 3).

The flow was solved via 3D incompressible BhatnagarGross-Krook D3Q19 LB methods ${ }^{33}$. We defined the nineteen discrete velocities by

$$
\begin{gathered}
\mathbf{e}_{\alpha}=(0,0,0), \alpha=0 \\
\mathbf{e}_{\alpha}=( \pm c, 0,0),(0, \pm c, 0),(0,0, \pm c), \alpha=1-6 \\
\mathbf{e}_{\alpha}=( \pm c, \pm c, 0),( \pm c, 0, \pm c),(0, \pm c, \pm c), \alpha=7-18
\end{gathered}
$$

where $c=\delta_{x} / \delta_{t}, \delta_{x}$ and $\delta_{t}$ are the lattice grid spacing and time step respectively $(=1)$. The evolution equation of the model was divided into two steps, the collision,

$$
\hat{f}_{\alpha}(\mathbf{x}, t)=\frac{1}{\tau}\left[f_{\alpha}(\mathbf{x}, t)-f_{\alpha}^{e q}(\mathbf{x}, t)\right]
$$

and the propagation,

$$
f_{\alpha}\left(\mathbf{x}+\mathbf{e}_{\alpha} \delta_{t}, t+\delta_{t}\right)=\hat{f}_{\alpha}
$$

where $f_{\alpha}$ is the density distribution function for particles moving with velocity $\mathbf{e}_{\alpha}$ at position $\mathbf{x}$ and time $t, \tau$ is the relaxation paramter, $\hat{f}$ is the density distribution function after collision and before propagation, $f_{\alpha}^{e q}$ is the equilibrium distribution function, and defined as

$$
\begin{gathered}
f_{0}^{e q}=1-\left(1-\omega_{0}\right) \frac{p}{c_{s}^{2}}+s_{0}(\mathbf{u}) \\
f_{\alpha}^{e q}=\omega_{\alpha} \frac{p}{c_{s}^{2}}+s_{\alpha}(\mathbf{u}), \alpha=1-18
\end{gathered}
$$

where $\mathbf{u}$ is the velocity, $p$ is the pressure, $s_{\alpha}(\mathbf{u})=$ $\omega_{\alpha}\left[3 \mathbf{e}_{\alpha} \cdot \mathbf{u}+4.5\left(\mathbf{e}_{\alpha} \cdot \mathbf{u}\right)^{2}-1.5 \mathbf{u}^{2}\right]$, with the weight coefficient $\omega_{0}=1 / 3, \omega_{1-6}=1 / 18, \omega_{7-18}=1 / 36$ and $c_{s}=c / \sqrt{3}$ is the speed of sound. The macroscopic flow velocity and pressure are computed from the distribution functions,

$$
\mathbf{u}=\sum_{\alpha=1}^{18} \mathbf{e}_{\alpha} f_{\alpha}, p=\frac{c_{s}^{2}}{1-\omega_{0}}\left[\sum_{\alpha=1}^{18} \mathbf{e}_{\alpha} f_{\alpha}+s_{0}(\mathbf{u})\right]
$$

The dynamic viscosity is given by

$$
\eta=c_{s}^{2}(\tau-1 / 2) \delta_{t}
$$

Shear-thinning effects were incorporated in the LB methods by calculating the local viscosity according to the local shear rate with the constitutive law of the fluid (equation 5), as usually done in LBM, see 343536 for details. The local shear rate $|\dot{\gamma}|$ is related to the second invariant of the rate-of-strain tensor $|\dot{\gamma}|=\sqrt{\varepsilon_{\alpha \beta} \varepsilon_{\alpha \beta}}$ that was calculated locally at each node by

$$
\varepsilon_{\alpha \beta}=-\frac{1}{2 c_{s}^{2} \tau \delta_{t}} \sum_{i=0}^{18} \mathbf{e}_{i \alpha} \mathbf{e}_{i \beta}\left[f_{i}(\mathbf{x}, t)-f_{i}^{e q}(\mathbf{x}, t)\right]
$$

The moving boundary conditions (imposed by the villi) were calculated by a computationnaly efficient combination of the bounce-back scheme ${ }^{32}$ and the transfer of momentum exerted on the fluid by the moving wall of velocity $\mathbf{u}_{w}$ (see 373839 for details) in the stair-case approximation

$$
f_{\bar{\alpha}}\left(\mathbf{x}_{l}, t+\delta_{t}\right)=\hat{f}_{\alpha}\left(\mathbf{x}_{l}, t\right)+6 \omega_{\alpha}\left(\mathbf{e}_{\alpha} \cdot \mathbf{u}_{w}\right)
$$

where $\mathbf{x}_{l}$ is fluid node such that $\mathbf{x}_{l}+\mathbf{e}_{\alpha}$ is a solid node, $\bar{\alpha}$ is the opposite direction of $\alpha$ (i.e. $\mathbf{e}_{\alpha}=-\mathbf{e}_{\bar{\alpha}}$ ), the $\hat{f}$ are taken after collision and before propagation and $f\left(., t+\delta_{t}\right)$ will be used at values after collision and after propagation. In the stair case approximation, this scheme is first order since bounceback implies that the surface has been artificially placed mid way between lattice nodes. Interpolation / extrapolation methods ${ }^{373839}$ allow to take into account the exact position of the boundary. These numerical schemes are second-order accurate but more computationally intensive in 3D. Preliminary tests showed that the first order scheme gave accurate results compared to the second order schemes and was kept for further simulations.

Null radial velocity components and pressures were imposed at the left and right of the model domain with the method of Guo et al. ${ }^{40}$. No-slip boundary conditions were imposed at the solid boundaries (figure 3). To simulate infinite arrays of villi in the Z-dimension, a periodic boundary condition was applied in this direction.

ADE were solved by one particular member of the family of 3D advection-diffusion D3Q7 LBM described by Ginzburg and called optimal advection ${ }^{41}$. Similarly, the algorithm is given (for $\alpha=0-6$ ) as

$$
\begin{gathered}
g_{\alpha}\left(\mathbf{x}+\mathbf{e}_{\alpha} \delta_{t}, t+\delta_{t}\right)=g_{\alpha}(\mathbf{x}, t)+\lambda\left[g_{\alpha}(\mathbf{x}, t)-g_{\alpha}^{e q}(\mathbf{x}, t)\right] \\
g_{\alpha}^{e q}=C\left(t_{\alpha}+\frac{1}{3} a^{(e)} t_{\alpha}^{(e)}+E_{\alpha}^{u}+\frac{1}{2} \mathbf{e}_{\alpha} \cdot \mathbf{u}\right) \\
C=\sum_{\alpha=0}^{6} \mathbf{e}_{\alpha} g_{\alpha}
\end{gathered}
$$

where $g$ is the density distribution function, $C$ the concentration, the collision eigenvalue $\lambda=-3+\sqrt{3}$, the weights $t_{0}=1 / 3$ and $t_{1-6}=1 / 6$, the basis vector $t_{0}^{(e)}=-3$ and $t_{1-6}^{(e)}=1 / 2$ and a velocity-dependent second-order correction 
to the diffusion tensor $E_{0}^{u}=-\mathbf{u}^{2}$ and $E_{1-6}^{u}=0.5\left(\mathbf{e}_{\alpha} \cdot \mathbf{u}\right)^{2}$. Finally, $a^{(e)}$ is connected to the diffusion coefficient $D$ via

$$
a^{(e)}=\frac{2 \sqrt{3} D}{c_{s}^{2} \delta_{t}}-1
$$

Instantaneous absorption by the villi was modeled via the Dirichlet boundary conditions in the stair-case approximation. Non-diffusive boundary conditions were used at the left and right of the domain ${ }^{32}$.

The communication and reconstruction of information at the grid transitions of the two grid sets of different sizes (between the coarse and fine grids) were done with the coupling algorithms of Dupuis and Chopard ${ }^{42}$ and Stiebler et al. ${ }^{43}$ for the flow and the advection-diffusion respectively, see ${ }^{4243}$ for details.

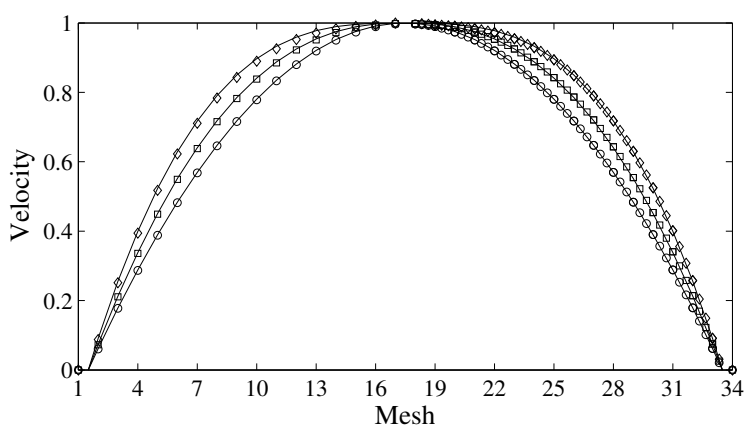

Fig. 4 Comparison of velocity profiles for a Poiseuille flow with power-law fluids with exact analytical solutions. The coarse mesh is on the left and the fine mesh on the right. Whatever the index $n$ is (diamond: $n=0.5$, box $: n=0.7$, circle $: n=1$ ), the numerical solution is in good agreement with the analytical solution and the velocity profile is continuous at the interface of the two grids.

These numerical methods were extensively validated in the literature 42414333 . The validity of the final code was checked using different benchmarks of similar flow problems. Firstly, an unsteady flow problem was tested by comparing the numerical solution with the analytical solution of the Womersley flow $^{44}$ on a domain of $30 \times 30 \times 30$ with periodic boundary conditions. The error $E=\left[\sum\left(u-u_{\text {exact }}\right)^{2} / \sum u_{\text {exact }}^{2}\right]^{1 / 2}$ was less than $3 \cdot 10^{-3}$ for Womersley numbers of 5 and 1 . Secondly, the effects of non-Newtonian rheology and the accuracy of the multi-grids algorithm was checked by the Poiseuille flow with power law fluids. The mesh used was the same than the one used for the applications with the villi. Figure 4 shows that the numerical solutions were in good agreement with the analytical solutions on the fine and coarse grids and the velocity was continuous at the interface of the two grids, whatever the value of $n$ was. Thirdly, the dispersion of a pulse, i.e. Taylor-Aris disperion $^{43}$, was used to validate the resolution of the ADE . The numerical solution was in excellent agreement with the analytical solution with and without advection $\left(E \leq 10^{-4}\right)$. These results lead us to have high confidence in our simulations.

Preliminary simulations were done to fix the different numerical parameters. The dimensions of the flow domain were chosen such that flow near the villi was not influenced by the boundary conditions at the top and the sides of the domain (figure 5). The height and the width of the whole computational domain was 2 and $3.4 \mathrm{~mm}$ respectively. For the fine grid, 53 nodes by millimeter were sufficient to obtain a high degree of accuracy. The comparison of the flow generated by groups of villi of different numbers has shown that five villi for the aligned villi configuration and nine villi (5 villi in the first row and 4 in the second) for the staggered configuration of villi were suitable to obtain representative results. In addition, based on estimates conducted in prior work ${ }^{19}$, greater numbers of villi would be more likely to extend onto the adjacent crests of the microfolds. The code was implemented with Matlab R2013a. For information, one single run required about thirty hours of simulations with a processor $2.7 \mathrm{GHz}$ Intel Core i5.

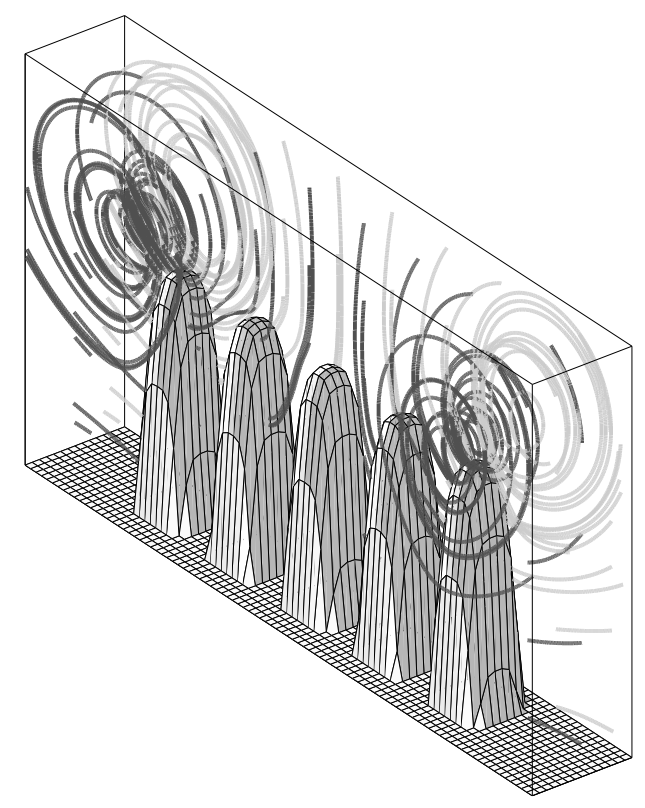

Fig. 5 Instantaneous streamlines around a group of villi of finger-like configuration during contraction or relaxation $(t=T / 2$ or $\mathrm{T} / 4$ ). The fluid is alternatively drawn into and pushed from the inter-villous space. Laminar eddies are present at the edges of the group of villi. The streamlines are qualitatively similar for a given row of villi in each of the three villous configurations. See figure 2 . 


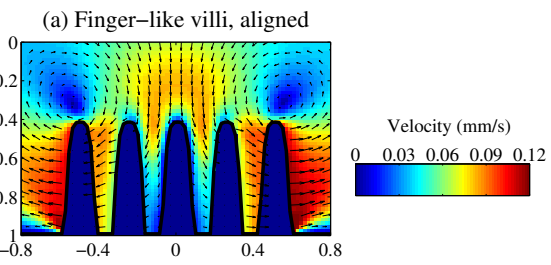

(b1) Finger-ligne villi, staggered $1^{\text {st }}$ row of villi

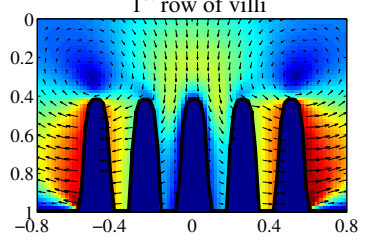

(c) Paddle-like villi, aligned

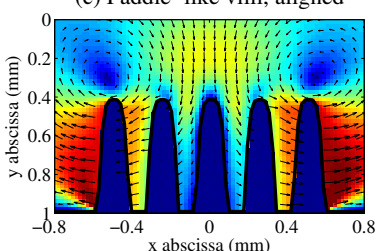

Fig. 6 Velocity fields during the relaxation $(t=T / 4)$ in the mid-plane $(\mathrm{X}, \mathrm{Y})$ of a row of villi with differing spatial dispositions and morphologies: aligned finger-like villi (a) ; staggered finger-like villi (b1 and b2); aligned paddle-like villi (c). See figure 2.

\section{Results}

\subsection{Characterisation of flow around villi}

The characteristics of three-dimensional flow around the villi were similar regardless of the shape and the spatial disposition of the villi (figure 2). The streamlines moved cyclically toward and away from the inter-villous space during the separation and the approximation of the villi respectively (figure 5). This action caused fluid to be drawn into the inter-villous region during the relaxation period (i.e. between 0 and $T / 2$ ) and ejected from it during contraction (i.e. between $T / 2$ and $T)$. Such flow resulted in the replacement of the fluid in the inter-villous space during each cycle of longitudinal contractile activity. There was only minor variation in the magnitude of flow around the mid-plane of the row of villi among the three configurations that were studied namely; aligned fingerlike villi, staggered finger-like villi and aligned paddle-like villi (figure 6 ). The Newtonian and the zero-shear viscosity $\eta_{0}$ had no impact on the flow: at the scale of the villi Reynolds number $R e=\rho \dot{\varepsilon}_{\text {max }} d^{2} / \eta_{0}$ are very low compared to the unity $\left(10^{-3}\right)$, where $\rho$ is the density $\left(10^{3} \mathrm{~kg} / \mathrm{m}^{3}\right)$ and $d$ the diameter of a villus $(160 \mu \mathrm{m})$, despite the low viscosity of the fluid around the villus (1.5 mPa.s $)^{20}$.

Two counter-rotating eddies were formed at each side of

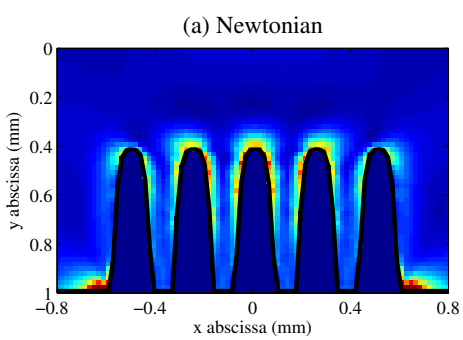

(b) Shear-thinning, $\mathrm{n}=0.6$

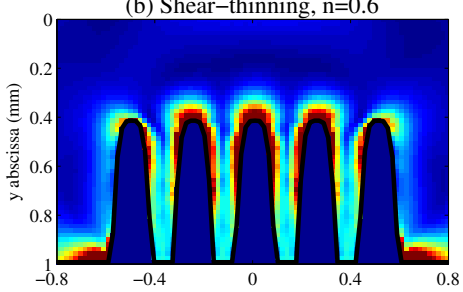

(c) Shear-thinning, $\mathrm{n}=0.4$
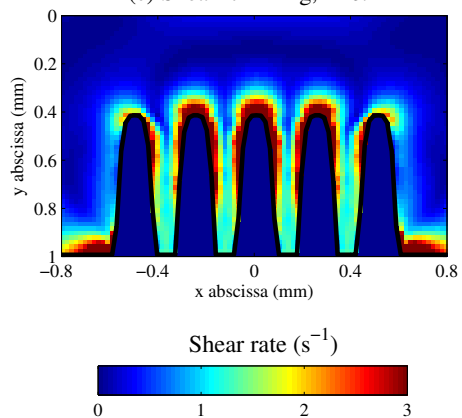

Fig. 7 Effects of varying degrees of shear-thinning (pseudoplasticity) on shear rate at time $T / 4$ of the cycle with finger-like aligned villi. The level of shear thinning influenced the levels of shear rate mainly in the inter-villous space notably when the critical shear rate $\dot{\gamma}_{c}$ was lower than 0.1 . a: Newtonian case, b: $n=0.6$ and $\dot{\gamma}_{c}=0.01$, c: $n=0.4$ and $\dot{\gamma}_{c}=0.01$.

the row of villi. Their directions of rotation alternating over the relaxation and contraction phases of the cycle of repeated longitudinal contraction (figures 5,6 ) and propagated in a direction perpendicular to the row of villi (i.e. along the $\mathrm{Z}$ axis). While the spatial disposition and the shape of the villi had only a minor impact on the flow, we found that a shear-thinning rheology in the fluid significantly altered the degree of shear in the inter-villous space with respect to that with a Newtonian fluid. Further, the shear rate in the inter-villous space increased when the index of the power-law was decreased (figure 7) but these effects operated only when the critical shear rate (equation 5) was lower than 0.1 , i.e. when the rheological behavior of the fluid tended to be strongly pseudo-plastic. Hence whilst the shear rate was about $0.5 \mathrm{~s}^{-1}$ in the inter-

6| Journal Name, 2010, [vol],1-10 
villous space with Newtonian fluids, it was increased by a factor of three when the values of $\dot{\gamma}_{c}\left(0.01 \mathrm{~s}^{-1}\right)$ and $n(0.4)$ were low. Also, the shear rate was maximum at the tips of the villi and did not propagated out of the inter-villous space. (a) High rate of diffusion
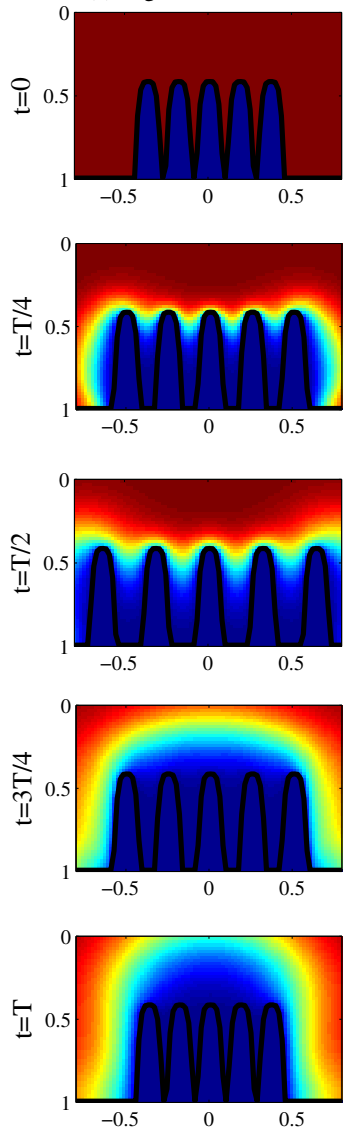

(b) Low rate of diffusion
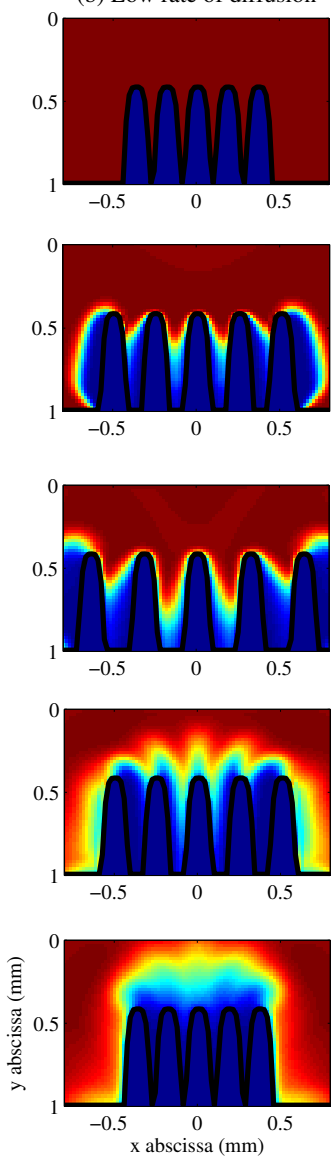

Fig. 8 Variation in the spatial disposition of molecular diffusive probes with high (a, $D=3.10^{-9} \mathrm{~m}^{2} / \mathrm{s}$ ) and low (b, $D=3.10^{-10}$ $\mathrm{m}^{2} / \mathrm{s}$ ) rates of diffusion in the mid-plane of a group of aligned finger-like villi during the course of a complete cycle of contractile activity $(T=6.66 \mathrm{~s})$. The probes were instantaneously absorbed by the villi. High values are red while low values are blue.

\subsection{Absorption and mixing around villi}

The flows generated by the approximation and separation of villi had a significant impact on absorption and mixing. In a situation where the concentration of nutrients in the flow domain was initially homogeneous (figure 8) nutrients were ab- sorbed following the entry of nutrient-rich fluid into the intervillous space during relaxation phase and a volume of fluid that was largely depleted of nutrients was ejected into the lumen during the contraction phase. For nutrients with high rates of diffusion $\left(D=3 \cdot 10^{-9} \mathrm{~m}^{2} / \mathrm{s}\right.$, e.g. glucose), absorption occurred predominantly at the tips of the villi, whereas for nutrients with low rates of diffusion $\left(D=3 \cdot 10^{-10} \mathrm{~m}^{2} / \mathrm{s}\right.$, e.g. biliary micelles), absorption took place over a greater proportion of the long axes of the villi. For low rate of diffusion, the gradient of concentration in the vertical direction has time to develop before to be absorbed by the villi. These phenomena were qualitatively independent of either the shape, the spatial disposition of the villi or the rheology of the fluid. Hence local micro-flows generated around villi by their horizontal movement enabled rapid turnover of the fluid in the inter-villous space, accelerating the process of absorption. This turnover extended on about $200-400 \mu \mathrm{m}$ above the tips of the villi (figure 8).

We also examined the effects of these local micro-flows on an initially horizontal plane of a diffusive tracer $(D=$ $3.10^{-10} \mathrm{~m}^{2} / \mathrm{s}$ ) placed above the tips of the villi (figure 9, $t=0$ ). It is noteworthy that the maximum dispersion of the diffusive tracer occurred in the inter-villous space (figure 9), where shear rates were the highest. Again the interposition of non-Newtonian rheology had a significant influence on dispersion in the inter-villous space. Hence the fluid in the inter-villous space was noticeably more dispersed with shearthinning ( $\dot{\gamma}_{c}=0.01 \mathrm{~s}^{-1}, n=0.4$ ) than with Newtonian fluids (figure 9), due to the larger shear rates that developed with the former (figure 7). The shear rate increase the local area between adjacent elements of fluids and so their inter-diffusion. The maximal concentration of the diffusive tracer that was observed in the inter-villous space $(t=3 T / 4)$ was $36 \%$ lower with the shear-thinning fluid than with the Newtonian fluid. The evolution of the absorbed mass during the cycle of concentration was also compared for the same situation (figure 10). Whatever the rheology of the fluids was, the movement of the villi increased the mass absorbed of one order of magnitude compared to the situation for which absorption was driven only by diffusion (i.e. villi are static). Shear-thinning behavior had significant effects when $\dot{\gamma}_{c} \geq 0.1$. As example, the absorption was increased of about $10 \%$ compared to the Newtonian situation when $n=0.4$ and $\dot{\gamma}_{c}=0.01$ (figure 10).

\section{Discussion and conclusion}

The results of this study showed that the approximation and subsequent separation of groups of villi, as observed during the formation of mucosal microfolds by periodic longitudinal contractions ${ }^{19}$, increased significantly mixing of digesta and absorption of nutrients adjacent to the mucosa i.e. in the perivillous space. These conclusions are based on the outcomes 


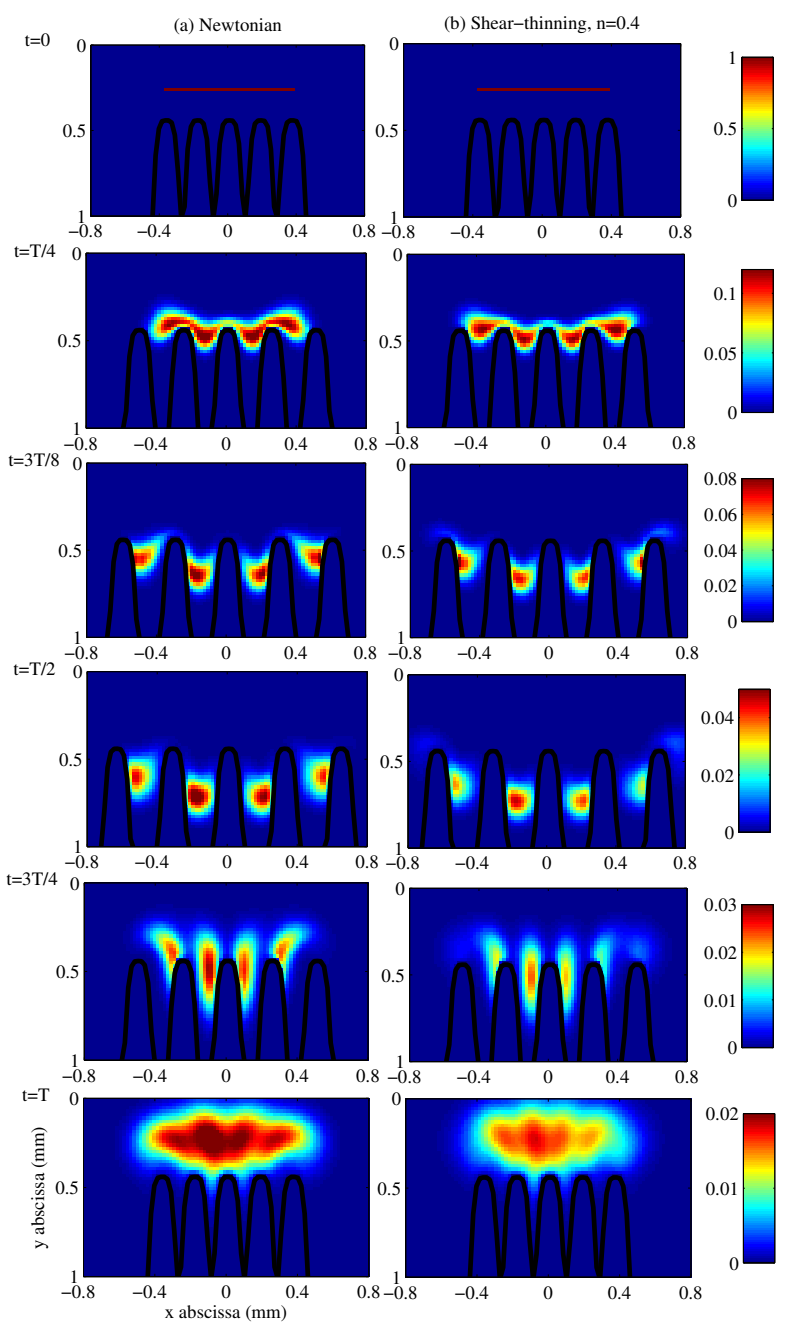

Fig. 9 Displacement of a diffusive probe $\left(D=3 \cdot 10^{-10} \mathrm{~m}^{2} / \mathrm{s}\right)$, initially aligned in a horizontal plane, during a contraction and relaxation cycle with finger-like aligned villi with (a) Newtonian and (b) shear-thinning $\left(n=0.4, \dot{\gamma}_{c}=0.01\right)$ fluids. Colour keys are the concentration.

of a 3D fluid mechanical model that incorporated the morphology, size and spacing of mucosal microfolds, that arise in the ex-vivo everted mucosa of the ileum of the possum during spontaneous longitudinal contractions ${ }^{19}$. It is noteworthy that micro-mixing resulting from mucosal folding does not require any intrinsic activity of the villi although it is possible that mixing may be augmented by intrinsic wavy or whip-like motions of villi 293031 .

In effect, micro-folds are generated by differences in the inherent mechanical properties of the component layers of the

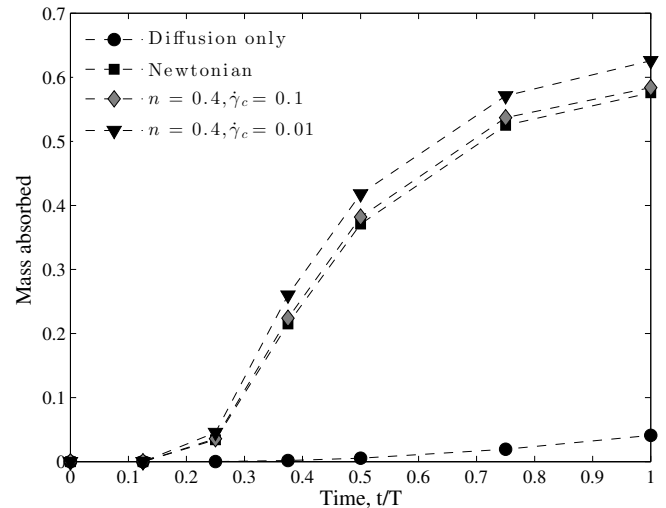

Fig. 10 Relative mass of a diffusive probe $\left(D=3 \cdot 10^{-10} \mathrm{~m}^{2} / \mathrm{s}\right.$, initially aligned in a horizontal plane, see figure 9) absorbed by the villi as a function of the time $t / T$ with Newtonian and shear-thinning fluids. The kinetics of absportion is also compared with the case where the villi are static and the absorption is only governed by diffusion (i.e. no flow conditions).

small intestine ${ }^{19}$ acting to augment mass transfer adjacent to the mucosa by generating mixing despite the high viscosity of the luminal content, and the low velocity of flow of digesta. Two processes achieve this mixing: the cyclic transfer of the fluid digesta between the inter-villous space and the periphery of the lumen and the formation of eddies at the margins of groups of villi (figure 5). The formation of such eddies was also predicted by a $2 \mathrm{D}$ generic multi-scale small intestinal flow model of Wang et al. ${ }^{26}$, that incorporated inherent contractile movements of groups of villi, albeit not based on firm quantitative data. These workers found that such movements induced vorticeal motions at the edges of each functional group of villi that enhanced the macro-scale advective flux ${ }^{26}$. The flow and mixing outcomes of the coupling of the micro- and macro- scales flows looks so to be an important feature of intestinal mixing. Our study focused on the role of microfolds on flow and mixing independently of external flow phenomena in an semi-infinite space. However, the interaction between the different scales could impact mixing and absorption at the periphery and in the center of the lumen. The second pattern of flow was the flow of digesta into and out of the inter-villous spaces. Not only this pattern accelerates the process of absorption by the periodic renewal of the fluid in the inter-villous space, it also generates sufficiently large amounts of shear that accelerates the dispersion of nutrients at the periphery of the lumen.

The fact that the patterns and extent of mixing did not depend upon the shape and the spatial disposition of the villi suggest that no evolutionary modification of the geometry or 
the spatial disposition of villi can act to increase mixing or mass transfers. The wide variation in the size and form of villi that occurs between regions of the small intestine and between species is thus more likely to result from corresponding differences in the rates of genesis and turnover of the component cells, notably the rates of division of the germ cell layer at the base and the loss of enterocytes from the villus tip, the latter possibly exacerbated by a greater abrasion from a high solid volume fraction of digesta or more abrasive particles in the $\operatorname{diet}^{45}$.

The digesta is described as an heterogeneous concentrated suspension $^{123}$. During the process of digestion, this suspension is transformed and exhibits different rheological properties: Newtonian, shear-thinning, pseudo-plastic, yield stress, etc. The influence of these rheological properties on the mechanics of mixing and more generally on the fluid mechanics of the gastro-intestinal tract is an open question. Our results show that peripheral mixing phenomena are more sensitive to pseudoplasticity (figures 7,9 ), than is mixing within the central lumen of the small intestine 4191516 because the levels of shear generated in the center of the lumen in absence of microfolds formation are too small to unveil pseudo-plastic behaviour. Hence regardless of the type of contractile activity that is taking place (peristalsis, segmentation, pendular activity), the maximum shear rate generated within the lumen of the small intestine is typically localised at the boundary and no more than $0.5 \mathrm{~s}^{-1}$, as shown by previous numerical simulations for Newtonian and non-Newtonian fluids 4191516 . Conversely our work showed that the shear rates in the intervillous space were almost an order of magnitude greater than this for pseudo-plastic fluids $\left(1-3 \mathrm{~s}^{-1}\right)$. Moreover pseudoplasticity increases the degree of shear and dispersion in the inter-villous space. Also, whilst longitudinal activity generates axial dispersion ${ }^{416}$, microfolds formation is a complementary mixing process that generate radial displacement of the digesta. Compared to the situation where mixing is only driven by diffusion, longitudinal activity increased dispersion of nutrients by a factor 2 after 4 min of activity ${ }^{4}$, whereas villi movements increased by an order of magnitude the mass absorbed of nutrients during one cycle of activity of 6 seconds. It is important to note that this work considered the role of villi movements on mixing and absorption for continuum media. However the digesta is multiphase fluid whose the elements can be of the same order of magnitude than the villi. For example, questions are open concerning the role of microfolds on the process of oil-water interface of dietary fat ${ }^{5}$.

Finally, we conclude that micro-folds formation is a major mixing mechanism in the small intestine and has the ability to augment mixing and absorption around the mucosa despite adverse rheology.

\section{References}

1 R. G. Lentle, Y. Hemar, C. E. Hall and K. J. Stafford, Journal of Comparative Physiology B-Biochemical Systemic and Environmental Physiology, 2005, 175, 337-347.

2 R. G. Lentle and P. W. M. Janssen, Journal of Comparative Physiology B-Biochemical Systemic and Environmental Physiology, 2008, 178, 673690.

3 J. Patarin, D. Bleses, A. Magnin, S. Guerin and C.-H. Malbert, Food Digestion, 2015, in Press.

4 C. de Loubens, R. G. Lentle, R. J. Love, C. Hulls and P. W. M. Janssen, Journal of the Royal Society Interface, 2013, 10, 20130027.

5 R. Lentle and C. de Loubens, Journal of Comparative Physiology B, 2015, 1-19.

6 R. G. Lentle, C. De Loubens, C. Hulls, P. W. M. Janssen, M. D. Golding and J. P. Chambers, Neurogastroenterology and Motility, 2012, 24, 68695.

7 P. W. Janssen and R. G. Lentle, Spatiotemporal Mapping Techniques for Quantifying Gut Motility. InNew Advances in Gastrointestinal Motility Research(pp. 219-241), Springer Netherlands, 2013.

8 D. L. Penry and P. A. Jumars, Bioscience, 1986, 36, 310-313.

9 D. L. Penry and P. A. Jumars, American Naturalist, 1987, 129, 69-96.

10 J. Engmann and A. S. Burbidge, Food and Function, 2013, 4, 443-447.

11 P. W. M. Janssen, R. G. Lentle, P. Asvarujanon, P. Chambers, K. J. Stafford and Y. Hemar, Journal of Physiology, 2007, 582, 1239-1248.

12 C. de Loubens, R. G. Lentle, C. Hulls, P. W. M. Janssen and R. J. Love, Plos One, 2014, 9, e95000.

13 J. Melville, E. Macagno and J. Christensen, American Journal of Physiology, 1975, 228, 1887-1892.

14 B. Jeffrey, H. S. Udaykumar and K. S. Schulze, American Journal of Physiology: Gastrointestinal and Liver Physiology, 2003, 285, 907-918.

15 R. J. Love, R. G. Lentle, P. Asvarujanon, Y. Hemar and K. J. Stafford, Food Digestion, 2013, 4, 26-35.

16 L. Fullard, W. Lammers, G. Wake and M. Ferrua, Food and Function, 2014, 5, 2731-42.

17 L. A. Fullard, W. J. Lammers and M. J. Ferrua, Journal of Food Engineering, 2015, 160, 1-10.

18 K. S. Schulze and E. Clark, Neurogastroenterology and Motility, 2008, 20, 1317-1327.

19 R. G. Lentle, P. W. M. Janssen, C. de Loubens, Y. F. Lim, C. Hulls and P. Chambers, Neurogastroenterology and Motility, 2013, 25, 881-E700.

20 Y. F. Lim, M. A. K. Williams, R. G. Lentle, P. W. M. Janssen, B. W. Mansel, S. A. J. Keen and P. Chambers, Journal of the Royal Society Interface, 2013, 10, 20121008.

21 Y. F. Lim, R. G. Lentle, P. W. Janssen, M. A. Williams, C. de Loubens, B. W. Mansel and P. Chambers, PloS one, 2014, 9, e100140.

22 V. L. Sallee and J. M. Dietschy, Journal of lipid research, 1973, 14, 47584.

23 H. Westergaard, K. H. Holtermuller and J. M. Dietschy, American Journal of Physiology, 1986, 250, G727-G735.

24 M. D. Levitt, A. Strocchi and D. G. Levitt, American Journal of Physiology, 1992, 262, G593-G596.

25 A. Strocchi, G. Corazza, J. Furne, C. Fine, A. DiSario, G. Gasbarrini and M. D. Levitt, American Journal of Physiology-Gastrointestinal and Liver Physiology, 1996, 270, G487-G491.

26 Y. Wang, J. G. Brasseur, G. Banco, A. G. Webb, A. C. Ailiani and T. Neuberger, Philosophical Transactions of the Royal Society Series B; Mathematical, Physical and Engineering Sciences, 2010, 368, 2863-2880.

27 E. L. Cussler, Diffusion: mass transfer in fluid systems, Cambridge university press, 2009.

28 R. E. Barry, Journal of Mammalogy, 1976, 57, 273-290.

29 B. Hambleton, American Journal of Physiology, 1914, 34, 446-447. 
30 C. King and L. Arnold, American Journal of Physiology, 1922, 59, 23.

31 W. A. Womack, J. A. Barrowman, W. H. Graham, J. N. Benoit, P. R. Kvietys and D. N. Granger, American Journal of Physiology, 1987, 252, G250-G256.

32 M. Sukop and D. Thorne Jr, Lattice Boltzmann Modeling: An Introduction for Geoscientists and Engineers, Springer, New York, ISBN 3540279814. URL edn, 2006, vol. 29.

33 R. Du and B.-c. Shi, Journal of Hydrodynamics, 2010, 22, 782-787.

34 S. P. Sullivan, L. F. Gladden and M. L. Johns, Journal of Non-Newtonian Fluid Mechanics, 2006, 133, 91-98.

35 Z. Chai, B. Shi, Z. Guo and F. Rong, Journal of Non-Newtonian Fluid Mechanics, 2011, 166, 332-342.

36 S. S. Mendu and P. K. Das, Journal of Non-Newtonian Fluid Mechanics, 2012, 175, 10-24.

37 M. Bouzidi, M. Firdaouss and P. Lallemand, Physics of Fluids, 2001, 13, 3452-3459.

38 P. Lallemand and L.-S. Luo, Journal of Computational Physics, 2003, 184, 406-421.

39 P.-H. Kao and R.-J. Yang, Journal of Computational Physics, 2008, 227, 5671-5690.

40 Z. L. Guo, C. G. Zheng and B. C. Shi, Chinese Physics, 2002, 11, 366 374.

41 I. Ginzburg, Advances in Water Resources, 2005, 28, 1171-1195.

42 A. Dupuis and B. Chopard, Physical Review E, 2003, 67, 066707.

43 M. Stiebler, J. Toelke and M. Krafczyk, Computers and Mathematics with Applications, 2008, 55, 1576-1584.

44 C. Loudon and A. Tordesillas, Journal of Theoretical Biology, 1998, 191, 63-78.

45 L. Montagne, J. R. Pluske and D. J. Hampson, Animal Feed Science and Technology, 2003, 108, 95-117. 